DAMTP

DAMTP-1999-85

University of Cambridge

UTAP-340

RESCEU-27/99

December 24, 2017

\title{
Stringy Probe Particle and Force Balance
}

\author{
Tetsuya Shiromizu \\ DAMTP, University of Cambridge \\ Silver Street, Cambridge CB3 9EW, United Kingdom \\ Department of Physics, The University of Tokyo, Tokyo 113-0033, Japan \\ and \\ Research Centre for the Early Universe(RESCEU), \\ The University of Tokyo, Tokyo 113-0033, Japan
}

\begin{abstract}
We directly derive the classical equation of motion, which governs the centre of mass of a test string, from the string action. In a certain case, the equation is basically same as one derived by Papapetrou, Dixon and Wald for a test extended body. We also discuss the force balance using a stringy probe particle for an exact spinning multi-soliton solution of Einstein-Maxwell-Dilaton-Axion theory. It is well known that the force balance condition yields the saturation of the Bogomol'nyi type bound in the lowest order. In the present formulation the gyromagnetic ratio of the stringy probe particle is automatically determined to be $g=2$ which is the same value as the background soliton. As a result we can confirm the force balance via the gravitational spin-spin interaction.
\end{abstract}

\section{INTRODUCTION}

Probe technique is useful for studying the approximate dynamics of multi-soliton systems as multi-black-holes. By the term of 'probe' we mean a sort of collective coordinate like the centre of mass. So-called test particle is one of probes and is governed by the geodesic equation. The test particle is frequently used to estimate the gravitational wave from binary systems in an early stage.

Among of the multi-soliton solution, BPS multi-soliton is an important object. The existence is guaranteed by the so-called force balance. The simplest example is the Majumdar-Papapetrou(MP) solution [i] in the $D=4$ Einstein-Maxwell theory or $N=2 D=4$ supergravity [2]. It is well known that the electrostatic force balances with the gravitational attractive force. Furthermore, the force balance still holds up to the gravitational spin-spin and dipole-spin interaction for the Israel-Wilson-Perjes(IWP) solution [3] which becomes the MP solution in the static limit, that is, the IWP solution is the spinning version of the MP solution [4]. The force balance condition yields the saturation of the Bogomol'nyi bound and the correct gyromagnetic ratio $g=2$ of a spinning charged test body 顿. We realise, however, that the same situation does not hold in the Einstein-Maxwell-Dilaton (EMD) system, that is, the force balance condition does not give the correct gyromagnetic ratio [5]. It was shown that the gyromagnetic ratio of the test body is different from that of the background space-time. This fact may indicate the non-existence of the spinning multi-soliton solution in the EMD system. On the other hand, we are aware of an exact solution of spinning multi-soliton in the $D=4$ Einstein-Maxwell-Dilaton-Axion (EMDA) system [6]. Obviously the new essential ingredient is the axion field. Hence, taking account of the contribution from the axion field is important to resolve the above discrepancy of the gyromagnetic ratio.

In this paper we derive the equation of motion(EOM) for the centre of mass of a string, say 'stringy probe particle', coupled with the general metric and the axion field. There may be two different ways to the end at first glance. One of them is the Papapetrou procedure [7] refined by Dixon [8] and Wald [9]. This approach is based on the local conservation low for the energy-momentum tensor and current of gauge fields. We expect that one can derive the EOM for the center of the mass of a string in this procedure although we will not show here. In this paper, we will adopt another approach which is expected to be simpler than the Papapetrou-Dixon-Wald procedure.

The $D=4$ EMDA system describes a low energy string theory. The low energy string effective action originally comes from the requirement of the conformal invariance on the two dimensional $\sigma$ model $[10]$. It tells us that we should back to the $\sigma$ model to derive the EOM for a stringy probe particle. The contribution from the electromagnetic 
and dilation field can be obtained by a certain dimensional reduction and conformal transformation from the string to the Einstein frame.

The rest of this paper is organised as follows. In Sec. II, we derive the EOM for a stringy probe beginning with the two-dimensional $\sigma$ model. The curved target space-time is ten dimensional. Through the adequate dimensional reduction to four dimensions we obtain the EOM in the string frame. In this formulation the gyromagnetic ratio of a probe is uniquely determined to be $g=2$. In Sec. III we will give an argument of the energy bound, so-called Bogomol'nyi type bound. In Sec. IV, we will discuss the force balance between a spinning exact solution of the $D=4$ EMDA system and a stringy probe particle via the gravitational spin-spin interaction. We confirm that the force balance holds when the Bogomol'nyi bound is saturated. Finally, we will give a summary and discussion in Sec. V.

\section{STRINGY PROBE PARTICLE}

\section{A. Stringy Probe Particle in Ten Dimensions}

The action of the string coupled with the general metric and axion field is

$$
S_{p}=-\frac{1}{2 \pi} \int d \tau d \sigma \sqrt{-\operatorname{det}\left(g_{a b}\right)}\left[G_{M N}(X) g^{a b}+\hat{B}_{M N}(X) \epsilon^{a b}\right] \partial_{a} X^{M} \partial_{b} X^{N}
$$

where $M=0,1, \ldots, 9$ and $a=0,1 . g_{a b}, G_{M N}$ and $\hat{B}_{M N}$ are the metric of the world volume of the string, the metric of the ten-dimensional space-time and the anti-symmetric tensor, respectively. In the above we omitted the dilaton term because of its small contribution. To obtain the effective action for the centre of the mass of a string, we decompose $X^{M}(\tau, \sigma)$ into the centre of mass and the excitation around it as follows,

$$
X^{M}(\tau, \sigma)=\bar{X}^{M}(\tau)+x^{M}(\tau, \sigma),
$$

where $\bar{X}^{M}(\tau):=(1 / \pi) \int_{0}^{\pi} d \sigma X^{M}(\tau, \sigma)$. Inserting Eq. (2.2) into Eq. 2.1 and integrating out of the coordinate $\sigma$, we obtain the effective action for $\bar{X}^{\mu}(\tau)$,

$$
\begin{aligned}
S_{p}= & \frac{1}{2} \int d \tau\left[G_{M N}(\bar{X}) \dot{\bar{X}}^{M} \dot{\bar{X}}^{N}+2 G_{M N}\left\langle\partial_{a} x^{M} \partial^{a} x^{N}\right\rangle+2 \partial_{I} G_{M N}(\bar{X}) \dot{\bar{X}}^{M}\left\langle x^{I} \dot{x}^{N}\right\rangle\right. \\
& \left.+\frac{1}{2} \partial_{I} \partial_{J} G_{M N}(\bar{X})\left\langle x^{I} x^{J}\right\rangle \dot{\bar{X}}^{M} \dot{\bar{X}}^{N}+2 \hat{B}_{M N}(\bar{X})\left\langle\dot{x}^{M} x^{N^{\prime}}\right\rangle+2 \partial_{I} \hat{B}_{M N}(\bar{X}) \dot{\bar{X}}^{M}\left\langle x^{I} x^{N^{\prime}}\right\rangle+\cdots\right] \\
= & \frac{1}{2} \int d \tau L_{\mathrm{eff}}(\bar{X}, \dot{\bar{X}}, t),
\end{aligned}
$$

where

$$
\langle F(\tau, \sigma)\rangle:=\frac{1}{\pi} \int_{0}^{\pi} d \sigma F(\tau, \sigma) .
$$

Here we assumed that the typical scale of the curvature of the background space-time is much larger than that of the typical scale of the string. For our present purpose it is enough that the excitation can be approximately described in the flat metric. The expression is given by

$$
x^{M}(\tau, \sigma)=\frac{i}{2} \sum_{n \neq 0}\left[\alpha_{n}^{M} e^{-2 i n(\tau-\sigma)}+\tilde{\alpha}_{n}^{M} e^{-2 i n(\tau+\sigma)}\right]
$$

for a closed string and is inserted into Eq. (2.3).

From the Euler-Lagrange equation for $\bar{X}^{\mu}$,

$$
\frac{\partial L_{\mathrm{eff}}}{\partial \bar{X}^{\mu}}-\partial_{\tau}\left(\frac{\partial L_{\mathrm{eff}}}{\partial \dot{\bar{X}}^{\mu}}\right)=0
$$

we obtain the formal EOM for the centre of the mass, 


$$
\begin{aligned}
\frac{D^{2} \bar{X}^{M}}{d \tau^{2}} & +\Gamma_{I J}^{M} n^{I J}+\frac{1}{2}\left(\partial_{I} \Gamma_{J N}^{M}-\partial_{N} \Gamma_{I J}^{M}\right) \dot{\bar{X}}^{J} \mathcal{S}^{I N}+\partial_{I} \Gamma_{J N}^{M} \dot{\bar{X}}^{J} N^{I N} \\
& -\frac{1}{2} \hat{H}_{I J}^{M} m^{I J}-\frac{1}{4}\left(\partial^{M} \hat{H}_{I J N}+\partial_{J} \hat{H}_{I N}^{M}\right) \dot{\bar{X}}^{J} \mathcal{T}^{I N}+\cdots=0,
\end{aligned}
$$

where $\hat{H}_{I J K}:=3 \partial_{[I} \hat{B}_{J K]}$ and

$$
\begin{gathered}
M^{I J}=\frac{i}{2} \sum_{n \neq 0} \frac{1}{n}\left(\alpha_{n}^{I} \tilde{\alpha}_{n}^{J}-\tilde{\alpha}_{n}^{I} \alpha_{n}^{J}\right) e^{-4 i n \tau}, \quad N^{I J}=\frac{i}{2} \sum_{n \neq 0} \frac{1}{n}\left(\alpha_{n}^{I} \tilde{\alpha}_{n}^{J}+\tilde{\alpha}_{n}^{I} \alpha_{n}^{J}\right) e^{-4 i n \tau} \\
m^{I J}=2 \sum_{n \neq 0}\left(\alpha_{n}^{I} \tilde{\alpha}_{n}^{J}-\tilde{\alpha}_{n}^{I} \alpha_{n}^{J}\right) e^{-4 i n \tau}, \quad n^{I J}=2 \sum_{n \neq 0}\left(\alpha_{n}^{I} \tilde{\alpha}_{n}^{J}+\tilde{\alpha}_{n}^{I} \alpha_{n}^{J}\right) e^{-4 i n \tau} \\
\mathcal{S}^{I J}=S^{I J}+\tilde{S}^{I J}, \quad \mathcal{T}^{I J}=-S^{I J}+\tilde{S}^{I J}+M^{I J} \\
S^{I J}=i \sum_{n=1} \frac{1}{n}\left(\alpha_{n}^{I} \alpha_{-n}^{J}-\alpha_{n}^{J} \alpha_{-n}^{I}\right), \quad \tilde{S}^{I J}=i \sum_{n=1} \frac{1}{n}\left(\tilde{\alpha}_{n}^{I} \tilde{\alpha}_{-n}^{J}-\tilde{\alpha}_{n}^{J} \tilde{\alpha}_{-n}^{I}\right)
\end{gathered}
$$

$S^{I J}$ and $\tilde{S}^{I J}$ are the angular momentum of the right-moving and left-moving excitations, respectively. For the later comparison we assume that the left-moving excitations is absent, that is,

$$
\tilde{\alpha}_{n}^{M}=0,
$$

and this implies

$$
n^{I J}=m^{I J}=M^{I J}=N^{I J}=\tilde{S}^{I J}=0 .
$$

This circumstance corresponds to that of the soliton which will be discussed later in Sec. IV.

As a result, Eq. (2.8) can be simplified in the form

$$
\frac{D^{2} \bar{X}^{M}}{d \tau^{2}}+\frac{1}{2}\left(\partial_{I} \Gamma_{J N}^{M}-\partial_{N} \Gamma_{J I}^{M}\right) \dot{\bar{X}}^{J} S^{I N}+\frac{1}{4}\left(\partial^{M} \hat{H}_{I J N}+\partial_{J} \hat{H}_{N I}^{M}\right) \dot{\bar{X}}^{J} S^{I N}+\cdots=0 .
$$

in ten dimensions. The second term may come from the ${ }^{(10)} R_{J I N}^{M} \dot{\bar{X}}^{J} S^{I N}$ and stands for the gravitational spin-spin interaction. The third term is just what we wanted and expresses the axion-spin interaction term occured due to the "additional ingredient", axion field. As we will show later, the term is essential for the force balance in the respect with the spin-spin interactions. We expect that the equation should be derived by the Papapetrou-Dixon-Wald approach too. Beside the axion term, the above equation is basically identical with the higher-dimensional version of one obtained in [7] [8] [9].

\section{B. Stringy Probe Particle in Four Dimensions}

Next, we derive the EOM for a stringy probe particle in four dimensions. To this end we now remember that the four dimensional low energy effective string action for the metric, electromagnetic, axion field and dilaton fields can be derived by a certain dimensional reduction of the ten dimensional low energy string action,

$$
S_{10}=\int d^{10} x e^{-2 \phi} \sqrt{-G}\left[R_{G}+4 G^{M N} \partial_{M} \phi \partial_{N} \phi-\frac{1}{12} G^{M N} G^{I J} G^{K L} \hat{H}_{M I K} \hat{H}_{N J L}\right] .
$$

The actual procedure is given by 11]

$$
G_{\mu \nu}=g_{S \mu \nu}+A_{\mu} A_{\nu}, \quad G_{4 \mu}=A_{\mu}, \quad G_{44}=1, \quad G_{a b}=\delta_{a b}, \quad G_{4 a}=G_{\mu a}=0
$$

and 


$$
\hat{B}_{\mu \nu}=B_{\mu \nu}, \quad \hat{B}_{4 \mu}=A_{\mu},
$$

where $\mu=0,1,2,3$ and $a=5,6,7,8,9$. In the above we imposed that all of the above fields do not depend on the coordinates $x^{4} \sim x^{9}$. We obtain the action in the string frame,

$$
S_{4}=\int d^{4} x e^{-2 \phi} \sqrt{-g_{S}}\left[R_{S}+4 g_{S}^{\mu \nu} \partial_{\mu} \phi \partial_{\nu} \phi-\frac{1}{12} e^{-4 \phi} g_{S}^{\mu \nu} g_{S}^{\alpha \beta} g_{S}^{\rho \sigma} H_{\mu \alpha \rho} H_{\nu \beta \sigma}-\frac{1}{2} e^{-2 \phi} g_{S}^{\mu \nu} g_{S}^{\alpha \beta} F_{\mu \alpha} F_{\nu \beta}\right],
$$

where $H_{\mu \nu \alpha}=3 \partial_{[\mu} B_{\nu \alpha]}+3 A_{[\mu} F_{\nu \alpha]}$. To obtain the action in the Einstein frame we have to take the conformal transformation as

$$
g_{S \mu \nu}=e^{2 \phi} g_{\mu \nu} .
$$

Then we obtain the action of the EMDA system,

$$
S_{4}=\int d^{4} x \sqrt{-g}\left[R-2 g^{\mu \nu} \partial_{\mu} \phi \partial_{\nu} \phi-\frac{1}{12} g^{\mu \nu} g^{\alpha \beta} g^{\rho \sigma} e^{-4 \phi} H_{\mu \alpha \rho} H_{\nu \beta \sigma}+\frac{1}{2} e^{-2 \phi} g^{\mu \nu} g^{\alpha \beta} F_{\mu \alpha} F_{\nu \beta}\right] .
$$

Now we can write down the EOM in terms of the four-dimensional quantities defined by Eqs. (2.13) and (2.14). In the string frame, the EOM for a stringy probe particle is immediately written as

$$
\begin{aligned}
\partial_{\tau}^{2} \bar{X}^{\mu}+{ }^{(4)} \Gamma_{\alpha \beta}^{\mu}(\bar{X}) \dot{\bar{X}}^{\alpha} \dot{\bar{X}}^{\beta}+\left(\dot{\bar{X}}^{4}+A_{\alpha} \dot{\bar{X}}^{\alpha}\right) \dot{\bar{X}}^{\nu} F_{\nu}{ }^{\mu}-\frac{1}{2} \partial^{\mu} F_{\alpha \beta}\left(\dot{\bar{X}}^{4}+A_{\rho} \dot{\bar{X}}^{\rho}\right) S^{\alpha \beta} \\
\quad+\frac{1}{2}\left(\partial_{\alpha}{ }^{(4)} \Gamma_{\nu \beta}^{\mu}-\partial_{\beta}{ }^{(4)} \Gamma_{\nu \alpha}^{\mu}\right) \dot{\bar{X}}^{\nu} S^{\alpha \beta}-\frac{1}{4}\left(\partial^{\mu} H_{\alpha \nu \beta}-\partial_{\nu} H_{\alpha \beta}^{\mu}\right) \dot{\bar{X}}^{\nu} S^{\alpha \beta}+O\left(\frac{1}{r^{5}}\right)=0 .
\end{aligned}
$$

In the above expression, we can show that $\dot{\bar{X}}^{4}+A_{\alpha} \dot{\bar{X}}^{\alpha}$ is approximately conserved. Noting the equation for $\bar{X}^{4}$,

$$
\partial_{\tau}^{2} \bar{X}^{4}-F_{\mu \nu} A^{\nu}\left(\dot{\bar{X}}^{4}+A_{\alpha} \dot{\bar{X}}^{\alpha}\right) \dot{\bar{X}}^{\mu}+{ }^{(4)} \nabla_{\mu} A_{\nu} \dot{\bar{X}}^{\mu} \dot{\bar{X}}^{\nu}=O\left(\frac{1}{r^{4}}\right)
$$

we can see that

$$
\partial_{\tau}\left(\dot{\bar{X}}^{4}+A_{\mu} \dot{\bar{X}}^{\mu}\right)=O\left(\frac{1}{r^{4}}\right)
$$

holds and we set

$$
\dot{\bar{X}}^{4}+A_{\mu} \dot{\bar{X}}^{\mu}=\frac{q}{m}+O\left(\frac{1}{r^{4}}\right)
$$

Finally we are resulted in

$$
\begin{aligned}
\partial_{\tau}^{2} \bar{X}^{\mu}+{ }^{(4)} \Gamma_{\alpha \beta}^{\mu}(\bar{X}) \dot{\bar{X}}^{\alpha} \dot{\bar{X}}^{\beta}+\frac{q}{m} \dot{\bar{X}}^{\nu} F_{\nu}{ }^{\mu}-\frac{q}{2 m} \partial^{\mu} F_{\alpha \beta} S^{\alpha \beta} \\
\quad+\frac{1}{2}\left(\partial_{\alpha}{ }^{(4)} \Gamma_{\nu \beta}^{\mu}-\partial_{\beta}{ }^{(4)} \Gamma_{\nu \alpha}^{\mu}\right) \dot{\bar{X}}^{\nu} S^{\alpha \beta}-\frac{1}{4}\left(\partial^{\mu} H_{\alpha \nu \beta}-\partial_{\nu} H_{\alpha \beta}^{\mu}\right) \dot{\bar{X}}^{\nu} S^{\alpha \beta}+O\left(\frac{1}{r^{5}}\right)=0
\end{aligned}
$$

in four dimensional string frame. Note that we can read from the above expression that the gyromagnetic ratio of the string probe particle is $g=2$. In the covariant form we may expect that the equation

$$
\frac{\mathcal{D}^{2} \bar{X}^{\mu}}{d \tau^{2}}-\frac{q}{m} F_{\nu}^{\mu} \dot{\bar{X}}^{\nu}-\frac{q}{2 m}{ }^{(4)} \nabla^{\mu} F_{\alpha \beta} S^{\alpha \beta}+\frac{1}{2}{ }^{(4)} R_{\nu \alpha \beta}^{\mu} \dot{\bar{X}}^{\nu} S^{\alpha \beta}-\frac{1}{4}\left({ }^{(4)} \nabla^{\mu} H_{\alpha \nu \beta}-{ }^{(4)} \nabla_{\nu} H_{\alpha \beta}^{\mu}\right) \dot{\bar{X}}^{\nu} S^{\alpha \beta}+\cdots=0
$$

holds, where $\mathcal{D} / d \tau:=\dot{\bar{X}}^{\mu}{ }^{(4)} \nabla_{\mu}$.

We can easily see that the dilaton field has no contributions to the leading order of the spin-spin interaction [5]. Since we are only interested in the force balance via the spin-spin interaction in this paper, we can go on in the string frame. If one wants to study the force balance in the Einstein frame, one should insert the above conformal transformation(Eq.(2.16)) into Eq. (2.22). It is, however, well known that the force balance between monopole components yields the saturation of a Bogomol'nyi bound $(|Q|=\sqrt{2} M$, in the present stringy case). 


\section{ENERGY BOUND FOR D=4 EINSTEIN-MAXWELL-DILATON-AXION THEORY}

In this section, we give the energy bound argument for the $D=4$ EMDA system in the Einstein frame. This is basically same as the proof given in Ref. [13] except for the existence of the axion field. In four dimensions the axion field can be expressed by a scalar field, $a$, as follows,

$$
H_{\mu \nu \alpha}=-e^{4 \phi} \epsilon_{\mu \nu \alpha \beta} \partial^{\beta} a .
$$

Here, bearing $N=4 D=4$ supergravity in mind, we define the supercovariant derivative for a spinor $\epsilon$ by

$$
\hat{\nabla}_{\mu} \epsilon=\nabla_{\mu} \epsilon-\frac{i}{4} e^{2 \phi} \partial_{\mu} a \epsilon+\frac{i}{4 \sqrt{2}} e^{-\phi} \gamma^{\rho \sigma} \gamma_{\mu} F_{\rho \sigma} \epsilon,
$$

where $\nabla_{\mu} \epsilon:=\left(\partial_{\mu}+\Gamma_{\mu}\right) \epsilon$ and $\Gamma_{\mu}$ is the spin connection,

$$
\Gamma_{\mu}=-\frac{1}{8} e^{\nu \hat{k}} \nabla_{\mu} e_{\nu}^{\hat{\ell}}\left[\gamma_{\hat{\ell}}, \gamma_{\hat{k}}\right] .
$$

In this section $\nabla_{\mu}$ stands for the covariant derivative associated with the Einstein metric $g_{\mu \nu}$. The spinor $\epsilon$ is assumed to satisfy the modified Witten equation $\gamma^{i} \hat{\nabla}_{i} \epsilon=0$. Furthermore, we define the Nester-like tensor [14],

$$
\begin{aligned}
\hat{E}^{\mu \nu} & :=\frac{1}{2}\left(\bar{\epsilon} \gamma^{\mu \nu \alpha} \hat{\nabla}_{\alpha} \epsilon-\overline{\hat{\nabla}_{\alpha} \epsilon} \gamma^{\mu \nu \alpha} \epsilon\right) \\
& =E^{\mu \nu}-\frac{i}{4} e^{2 \phi} \partial_{\alpha} a \bar{\epsilon} \gamma^{\mu \nu \alpha} \epsilon-\frac{i}{\sqrt{2}} e^{-\phi} \bar{\epsilon}\left(F^{\mu \nu}-\gamma_{5} \tilde{F}^{\mu \nu}\right) \epsilon,
\end{aligned}
$$

where $\tilde{F}^{\mu \nu}=(1 / 2) \epsilon^{\mu \nu \alpha \beta} F_{\alpha \beta}$. Hereafter we assume that $\epsilon$ has the chirality of $\gamma_{5} \epsilon=i \epsilon$. This is influenced by $N=4$ $D=4$ supergravity. The divergence of the Nester-like tensor becomes

$$
\begin{aligned}
\nabla_{\nu} \hat{E}^{\mu \nu}= & \frac{1}{2} G_{\nu}^{\mu} V^{\nu}+\overline{\hat{\nabla}_{\alpha} \epsilon} \gamma^{\mu \nu \alpha} \hat{\nabla}_{\beta} \epsilon-\frac{1}{2} T_{\nu}^{\mu}(F) V^{\nu}-\frac{1}{\sqrt{2}} e^{\phi} \nabla_{\nu}\left(e^{-2 \phi} F^{\mu \nu}\right) \bar{\epsilon} \epsilon \\
& -\frac{i}{2} \partial_{\nu} \phi \partial_{\alpha} a e^{2 \phi} \bar{\epsilon} \gamma^{\mu \nu \alpha} \epsilon-\frac{i}{\sqrt{2}} e^{\phi} \partial_{\nu} \phi \bar{\epsilon}\left(F^{\mu \nu}+\gamma_{5} \tilde{F}^{\mu \nu}\right) \epsilon,
\end{aligned}
$$

where

$$
T_{\mu \nu}(F)=e^{-2 \phi}\left(F_{\mu \rho} F_{\nu}^{\rho}-\frac{1}{4} g_{\mu \nu} F^{2}\right) .
$$

After some tedious calculations we obtain

$$
\nabla_{\nu} \hat{E}^{\mu \nu}=T_{\nu}^{\mu}(\text { mat }) V^{\nu}+\overline{\hat{\nabla}_{\alpha} \epsilon} \gamma^{\mu \alpha \beta} \hat{\nabla}_{\beta} \epsilon-\overline{\delta \lambda} \gamma^{\mu} \delta \lambda+\frac{i}{\sqrt{2}} \bar{\epsilon} J^{\mu} \text { (mat) } \epsilon,
$$

where $V^{\mu}=\bar{\epsilon} \gamma^{\mu} \epsilon$ and $\delta \lambda$ is defined by

$$
\delta \lambda=\frac{1}{\sqrt{2}}\left(\gamma^{\mu} \partial_{\mu} \phi+\frac{1}{2} e^{2 \phi} \gamma^{\mu} \gamma_{5} \partial_{\mu} a-\frac{i}{2 \sqrt{2}} \gamma^{\mu \nu} F_{\mu \nu}\right) \epsilon .
$$

One can interpret the field $\lambda$ as the dilatino which is a superpartner of the dilation field in supergravity. In the derivation of Eq. (3.7) we used the field equations

$$
G_{\mu \nu}=2 T_{\mu \nu}(\phi)+2 T_{\mu \nu}(a)+2 T_{\mu \nu}(F)+2 T_{\mu \nu}(\text { mat })
$$

and

$$
\nabla_{\nu}\left(e^{-2 \phi} F^{\mu \nu}\right)+\frac{1}{2} \tilde{F}^{\mu \nu} \partial_{\nu} a=-J^{\mu}(\mathrm{mat})
$$

where

$$
T_{\mu \nu}(\phi)=\partial_{\mu} \phi \partial_{\nu} \phi-\frac{1}{2} g_{\mu \nu} \partial^{\rho} \phi \partial_{\rho} \phi
$$


and

$$
T_{\mu \nu}(a)=\frac{e^{4 \phi}}{4}\left(\partial_{\mu} a \partial_{\nu} a-\frac{1}{2} g_{\mu \nu} \partial^{\rho} a \partial_{\rho} a\right) .
$$

We also used the following expression,

$$
\begin{aligned}
\overline{\delta \lambda} \gamma^{\mu} \delta \lambda= & T_{\nu}^{\mu}(\phi) V^{\nu}-T_{\nu}^{\mu}(a) V^{\nu}-\frac{1}{2} T_{\nu}^{\mu}(F)-\frac{1}{2} e^{2 \phi} \partial_{\nu} \phi \partial_{\alpha} a \bar{\epsilon} \gamma^{\nu \mu \alpha} \gamma_{5} \epsilon \\
& +\frac{i}{\sqrt{2}} e^{-\phi} \partial_{\nu} \phi \bar{\epsilon}\left(F^{\mu \nu}+\gamma_{5} \tilde{F}^{\mu \nu}\right) \epsilon+\frac{i}{2 \sqrt{2}} e^{-\phi} \partial_{\nu} a \bar{\epsilon}\left(F^{\mu \nu}+\gamma_{5} \tilde{F}^{\mu \nu}\right) \gamma_{5} \epsilon
\end{aligned}
$$

Integrating Eq. (3.7) over the full space volume $V$ and assuming the dominant energy condition, we obtain the inequality

$$
\begin{aligned}
\int_{S_{\infty}} d S_{\mu \nu} \hat{E}^{\mu \nu} & =\int_{V} d \Sigma_{\mu} \nabla_{\nu} \hat{E}^{\mu \nu} \\
& =\int_{V} d \Sigma_{\mu}\left[T_{\nu}^{\mu}(\text { mat }) V^{\nu}+\overline{\hat{\nabla}_{\alpha} \epsilon} \gamma^{\mu \alpha \beta} \hat{\nabla}_{\beta} \epsilon-\overline{\delta \lambda} \gamma^{\mu} \delta \lambda+\frac{i}{\sqrt{2}} \bar{\epsilon} J^{\mu}(\text { mat }) \epsilon\right] \geq 0 .
\end{aligned}
$$

The left hand-side in the first line of the above can be written in the term of several conserved charges, ADM four-momentum $P_{\mathrm{ADM}}^{\mu}$, the electric charge $Q$ and magnetic charge $P$, as follows [12,

$$
\int_{S_{\infty}} d S_{\mu \nu} \hat{E}^{\mu \nu}=\bar{\epsilon}_{0} \gamma_{\mu} \epsilon_{0} P_{\mathrm{ADM}}^{\mu}-\frac{1}{\sqrt{2}} \bar{\epsilon}_{0}\left(Q-\gamma_{5} P\right) \epsilon_{0},
$$

where $\epsilon_{0}$ is the constant spinor which is identical to the limit value of $\epsilon$ at the spatial infinity. Hence we obtain the Bogomol'nyi type bound,

$$
M \geq \frac{1}{\sqrt{2}} \sqrt{Q^{2}+P^{2}}
$$

In the absence of the magnetic charge, $P=0$, the saturation of the bound occurs as $M=(1 / \sqrt{2})|Q|$.

\section{FORCE BALANCE VIA GRAVITATIONAL SPIN-SPIN INTERACTION}

In this section we will confirm the force balance between the gravitational spin-spin, dipole-spin and axion-spin interactions when the Bogomol'nyi type bound is saturated. As we said in Sec. I, the spinning multi-soliton solution exists in the $D=4$ EMDA system. This solution is the straightforward extension of the IWP solution and saturates the Bogomol'nyi bound, i.e., $|Q|=\sqrt{2} M$. We expect that the force balance holds via the gravitational spin-spin interaction therein. To check the force balance, we consider the motion of a stringy probe particle on the background geometry of the single soliton with $Q=\sqrt{2} M$. In the Einstein frame the single solution is given by [6]

$$
d s^{2}=-e^{2 \phi}\left(d t+\omega_{i} d x^{i}\right)^{2}+e^{-2 \phi} d \mathbf{x}^{2}
$$

and

$$
A=\frac{1}{\sqrt{2}} e^{2 \phi}\left(d t+\omega_{i} d x^{i}\right)
$$

where $i=1,2,3$ and $\epsilon^{i j k} \partial_{j} \omega_{k}=-\partial_{i} a$. In the limit of the slow rotating the metric, vector potential and axion field are written as

$$
\begin{gathered}
d s^{2}=-\left(1+\frac{2 M}{r}\right)^{-1} d t^{2}+\left(1+\frac{2 M}{r}\right) d \mathbf{x}^{2}+\frac{4 \epsilon_{i j k} J^{j} x^{k}}{r^{3}} d t d x^{i}+O\left(\alpha^{2}\right) \\
A \simeq \frac{1}{\sqrt{2}} e^{2 \phi} d t-\frac{Q}{r^{3} M} \epsilon_{i j k} J^{j} x^{k} d x^{i}
\end{gathered}
$$


and

$$
a=\frac{2 J^{i} x^{i}}{r^{3}}+O\left(\alpha^{2}\right)
$$

where $\alpha$ is the parameter of the angular momentum so that $\vec{J}=(0,0, M \alpha)$. One can find the exact expression of the above solution in Ref. [6].

In the order of $O\left(1 / r^{3}\right)$, the spatial components of the equation of the motion for a probe is given by

$$
\begin{aligned}
\partial_{\tau}^{2} \bar{X}^{i} & \simeq{ }^{(4)} \Gamma_{\alpha \beta}^{i} \dot{\bar{X}}^{\alpha} \dot{\bar{X}}^{\beta}+\frac{q}{m} F_{\alpha}^{i} \dot{\bar{X}}^{\alpha} \\
& \simeq-{ }^{(4)} \Gamma_{00}^{i}+\frac{q}{m} F_{0}^{i} \\
& =\frac{1}{2} g_{S}^{i j} \partial_{j} g_{S 00}+\frac{q}{m} \partial^{i} A_{0} \\
& \simeq-\left(2-\sqrt{2} \frac{q}{m}\right) \partial^{i} \phi
\end{aligned}
$$

because of $g_{S 00}=e^{2 \phi} g_{00}=-e^{4 \phi}$. Hence we can see the force cancellation between the Newtonian-like gravitational and the Coulombic electrostatic forces when $q=\sqrt{2} m$ holds.

Let us confirm the force balance via the gravitational spin-spin interaction. The spin-spin, dipole-spin and axion-spin interaction forces are

$$
\begin{gathered}
F_{\text {spin }}^{i}=-\frac{1}{2} R_{0 j k}^{i} S^{j k}+O\left(\frac{1}{r^{5}}\right) \\
F_{\text {dipole }}^{i}=\frac{q}{2 m} S^{j k(4)} \nabla^{i} F_{j k}+O\left(\frac{1}{r^{5}}\right)
\end{gathered}
$$

and

$$
F_{\text {axion }}^{i}=-\frac{1}{4} \partial^{i} H_{j 0 k} S^{j k}+O\left(\frac{1}{r^{5}}\right)
$$

respectively. Thus the total force in the order of $O\left(1 / r^{4}\right)$ becomes

$$
F_{\text {spin }}^{i}+F_{\text {dipole }}^{i}+F_{\text {axion }}^{i}=\left(2-\frac{q Q}{m M}\right) \partial_{i}\left[\frac{-\mathbf{J} \cdot \mathbf{S}+3(\mathbf{S} \cdot \hat{\mathbf{r}})(\mathbf{J} \cdot \hat{\mathbf{r}})}{r^{3}}\right]+O\left(\frac{1}{r^{5}}\right),
$$

where $S^{i}:=(1 / 2) \epsilon^{i j k} S^{j k}$ and $\hat{\mathbf{r}}:=\mathbf{r} / r$. As we can easily see, the above total force exactly vanishes when the Bogomol'nyi type bound is saturated, that is, $Q=\sqrt{2} M$ and $q=\sqrt{2} m$.

\section{SUMMARY AND DISCUSSION}

In this paper we derived the equation of motion for a stringy probe particle coupled with the axion field. Its gyromagnetic ratio is automatically determined in the present formulation and turns out to be $g=2$. Moreover, we confirmed that the force balance holds via the gravitational spin-spin interaction when the Bogomol'nyi type bound is saturated. The behaviour of the probe seems to be related to the existence of the multi-soliton solution in the $D=4$ Einstein-Maxwell-Dilaton-Axion(EMDA) system.

We investigated the force balance in four dimensions. As we can see the multi-soliton solution has naked singularity in the $D=4$ EMDA system [6] as well as the Kerr-Newman space-time at the saturation of the Bogomol'nyi bound. On the other hand, Horowitz and Sen found a multi-soliton solution without naked singularity in $D>5$-dimension [15]. They also made the four dimensional black hole space-time by taking doubly periodic allays in six dimensions. So the force balance therein is also important and it seems to be easy to extend our present work to the higher-dimensional version.

As we moderately said, the value of the gyromagnetic ratio is important to discover exact spinning multi-soliton solutions. Before we try to discover it is advisable to check the value of probe gyromagnetic ratio, that is, whether the value gyromagnetic ratio is same as that of the background soliton or not. If same, we are able to have a great chance to discover a new solution. We think that this phrase may be valid for higher-dimensional objects like D-brane [16] 
[17. In addition, one might be going to find an exact solution of the cosmological spinning multi-black hole which should be the extension of the cosmological non-spinning multi-black hole exact solution discovered by Kastor and Traschen 18. So, we should check the force balance in cosmos.

Our basic equation (Eq. (2.22)) might be useful to study the approximate dynamics of a test string in a curved background space-time. Taking account of the axion terms is essential if the string is excited. If the string stays in the ground state, the motion is simply geodesic one. In our approach, a test string is assumed to be widely separated from the central soliton. If not so, we cannot use the excitation form of Eq. (2.6) in the flat metric and must consider the time evolution due to the strong gravity or tidal forces [19].

Finally, we should give a comment on the assumption of Eq. (2.9). From the analysis given in Sec. IV, we realise that the stringy probe particle plays as the test body like the central soliton under the assumption. Namely, the soliton given by Eqs. (4.1) and (4.2) seems to be related to the right-moving string state. This is because the solution is in the BPS state and the absence of the left(or right) moving excitation(oscillation) is essential for the BPS state [20].

Beside our present purpose of the force balance, Eq. (2.8) should be used for general cases having both excitations. The comparison with the equation derived by the Papapetrou procedure [7] is important because it is not likely that the extra terms does not appear in the procedure.

\section{Acknowledgment}

The author is grateful to Gary Gibbons and DAMTP relativity group for their hospitality. He also thanks M. Spicci for his careful reading of this manuscript. This study is supported by JSPS(No. 310).

[1] S. D. Majumdar, Phys. Rev. 72, 930(1947)

A. Papapetrou, Proc. Roy. Irish Acad.A51, 191(1947)

[2] K. P. Tod, Phys. Lett. B121, 241(1981)

[3] Z. Perjes, Phys. Rev.Lett.27, 1668(1971)

W. Israel and G. A. Wilson, J. Math. Phys.13, 865(1972)

[4] J. Tiomno, Phys. Rev. D7, 356(1973);

W. Israel and J. T. J. Spanos, Lett. Nouvo Cim. 7, 245(1973);

D. Kastor and J. Traschen, Class. Quantum Grav. 16, 1265(1999)

[5] T. Shiromizu, to be published in Phys. Lett. B(1999), hep-th/9906177

[6] R. Kallosh, D. Kastor, T. Ortin and T. Torma, Phys. Rev. D50, 6374(1994);

E. Bergshoeff, R. Kallosh and T. Ortin, Nucl. Phys. 478, 156(1996)

[7] A. Papapetrou, Proc. Roy. Soc. A209, 248(1951)

[8] W. G. Dixon, Phil. Trans. Roy. Soc. 277A, 59(1960);

Proc. Roy. Soc. Lond. A314, 499(1970)

[9] R. M. Wald, Phys. Rev. D6, 406(1972)

[10] C. G. Callan, D. Friedan, E. J. Martinec and M. J. Perry, Nucl. Phys. B262, 593(1985)

[11] E. Bergshoeff, R. Kallosh and T. Ortin, Phys. Rev. D50, 5188(1994)

[12] For the typical example in the Sec. IV, the axionic charge does not affect the bound. In fact, the axionic charge term becomes $\sim \int d S_{\mu \nu} \epsilon^{\mu \nu \alpha \beta} \partial_{\alpha} a \bar{\epsilon}_{0} \gamma_{\beta} \epsilon_{0} \sim \int d S\left(x^{i} / r\right) \epsilon^{0 i j k} \partial_{j} a \bar{\epsilon}_{0} \gamma_{k} \epsilon_{0}=0$.

[13] G. W. Gibbons, D. Kastor, L. A. J. London, P. K. Townsend and J. Traschen, Nucl. Phys. B416, 850(1994)

[14] J. Nester, Phys. Lett. A83, 241(1981)

[15] G. T. Horowitz and A. Sen, Phys. Rev. D53, 808(1996)

[16] J. Polchinski, Phys. Rev. Lett., 75, 4724(1995)

[17] A. A. Tseytlin, Nucl. Phys. B487, 141(1997);

M. Cevetic and D. Youm, Nucl. Phys. B499, 253(1997);

V. Balasubramanian, D. Kastor, J. Traschen and K. Z. Win, Phys. Rev. D59, 084007(1999)

[18] D. Kastor and J. Traschen, Phys. Rev. D47, 5370(1993)

[19] G. T. Horowitz and A. R. Steif, Phys. Rev. D42, 1950(1990);

G. T. Horowitz and S. F. Ross, Phys. Rev. D57, 1098(1998)

[20] For example, J. M. Maldacena, PhD Thesis, hep-th/9607235 